\title{
Evaluation of clinical and Para clinical findings, treatment response in RA patients: Data from a single center RA registry
}

\author{
Sara Jalali-Jivan ${ }^{1}$, Shafieh Movassaghi ${ }^{1 *}$, Seyed Reza Najafizadeh ${ }^{1}$, Abdolrahman Rostamian ${ }^{1,2}$, Rabeah Rajabzadeh ${ }^{1}$, \\ Hengameh Babaei-Lakeh ${ }^{3}$, Mahroo Mohammad Hoseini ${ }^{3}$, Amirmasoud Kazemzadeh-Houjaghan ${ }^{4}$, Ghazal Behzad ${ }^{4}$ \\ ${ }^{1}$ Department of Rheumatology, Imam Khomeini Hospital, School of Medicine, Tehran University of Medical Sciences, \\ Tehran, Iran. ${ }^{2}$ Rheumatology Research Center, Imam Khomeini Hospital, Tehran University of Medical Sciences, Tehran, \\ Iran. ${ }^{3}$ School of Medicine, Islamic Azad University of Medical science, Tehran, Iran. ${ }^{4}$ School of Medicine, Tehran \\ University of Medical Sciences, Tehran, Iran.
}

\begin{abstract}
Rheumatoid arthritis (RA) is a chronic, inflammatory autoimmune disease characterized by the inflammatory arthritis rheumatoid factor (RF: An IgM produced against anti-immunoglobulin G) and anti-cyclic citrullinated peptide (anti-CCP) autoantibodies. In the present study, the aim was to register RA patients in Imam Khomeini Hospital, Tehran, Iran.

The study population of this research was RA patients referred to the rheumatology clinic in 2019 at Imam Khomeini Hospital in Tehran and had been diagnosed 5 years ago. Demographic, clinical, and laboratory information of patients at the beginning of referral at 3 months, and 1, 2, 3, and 4 years after referral, were carefully recorded in each patient questionnaire.

In the first visit, 79 RA patients were examined and in the other 5 visits, 73, 52, 33, 20, 13 patients, respectively, were examined totaling the 270 visits. RF and anti-CCP were positive in $58.22 \%$ and $62.02 \%$ of patients, respectively. The mean age of patients was $52.5 \pm 11.25$ years. The results of the present study showed that the mean morning stiffness, DAS, arthritis, arthralgia, ESR, and CRP decreased from the second visit through the following five visits which indicated disease control.

Clinical and laboratory data showed considarable disease control, however, relapse of disease was seen due to drug discontinuation. Registering the disease makes it possible to record the necessary information of each patient, including demographic, clinical, and paraclinical findings as well as consultations, and gives the physician accurate access to patient information and unnecessary duplication of paraclinical examinations.
\end{abstract}

Keywords: Rheumatoid arthritis; Registry

\section{Introduction}

Rheumatoid arthritis (RA) is a systemic autoimmune disease with a prevalence of $0.1 \%$ to $1.9 \%$ of the adult population that affects women 2-3 times more often than men and is characterized by synovial inflammation, cartilage, and bone destruction [1, 2]. Therapeutic approaches in RA include non-steroidal anti-inflammatory drugs (NSAIDs), disease-modifying anti-rheumatic drugs (DMARDs) such as methotrexate, biologic agents such as TNF- $\alpha$ inhibitors, and corticosteroids [3].

Disease Registration is an organized system for collecting, storing and retrieving, analyzing, and disseminating information about a person with a specific disease or is exposed to the known or suspected adverse effects in a population and a certain geographical area [4]. One of the most important goals of the disease registration program is to investigate the incidence and prevalence of the disease, as well as evaluation of the disease, quality of patient care services, measure and monitoring the safety and injury, and even promote research projects. The importance of registering diseases in research includes survival analysis and evaluation of clinical care outcome, etiological studies, production of descriptive information (incidence of mortality), economic and managerial analysis, and a suitable source for clinical trial, case, and cohort studies [5]. It should be noted that disease registration is considered as one of the practical pillars of public health [6]. Implementation of a registry system for RA disease can help to obtain accurate statistics of the incidence, type of treatment approach, and also reduce its complications. In Iran, Rheumatry is the Iranian 
rheumatology registry that was established in 2016. The rheumatry.com web site is an online registry system.

According to previous studies, implementation of a registry system for RA is useful to study the long-term effects of RA treatment and complications, the survival rate, and cause of death of RA patients. For example, the register of RA patients in France has shown that RA patients receiving biologics have a higher risk of infections and lymphomas [7]. Another study based on the RA patient registry in France showed that RA patients who are positive for anti-CCP respond better to Abatacept [8]. The important role of the disease registry in diagnosis, treatment, and follow-up of the patients is undeniable. Unfortunately, no study has been reported in Iran to register RA patients. In the present study, our aim was to report the clinical and paraclinical findings and treatment response in RA patients from a single center RA registry.

\section{Materials and Methods}

\section{Study population}

In this retrospective cross-sectional study, the study population included RA patients referred to the rheumatology outpatient clinic of Imam Khomeini Hospital, University of Medical Sciences, Tehran, Iran, who were diagnosed 5 years ago. Inclusion criteria was: Diagnosis of RA disease based on the 2010 criteria of American College Rheumatology (ACR) [9], informed consent of the patient for participation in the study, and adults over 18 years. Exclusion criteria were patient dissatisfaction of participation in the project.

Demographic information of patients including age, sex, age of onset of disease, disease duration, coexistence of other diseases, and occupation was recorded for each patient. Laboratory findings were carefully recorded on the first visit, 3 to 6 months later, and then annually in each patient questionnaire including complete blood count (CBC), calcium, phosphorus, creatine, anti-nuclear antibody (ANA), florescent anti-nuclear antibody (FANA), rheumatoid factor (RF), C-reactive protein (CRP), erythrocyte sedimentation rate (ESR), anti-cyclic citrullinated peptide (anti-CCP), aspartate amino transferase (AST), alanine amino transferase (ALT), creatine phosphokinase (CPK), and lactate dehydrogenase (LDH)), as well as clinical manifestations (morning stiffness, joint pain, joint thunder, and joint effusion), and of course, prognosis (response to treatment and the progression of the disease). Disease activity score 28 (DAS 28) was used to evaluate the disease activity, then, all data were entered on the rheumatry.ir ${ }^{1}$ website. The protocol of the study was approved by the Ethical Committee of Tehran University of Medical Sciences (Code: IR.TUMS .IKHC.REC.1398.244).

\section{Statistical Analysis}

Data analysis was performed to describe the demographic and clinical status of patients using SPSS software (version 25) at both the descriptive and analytic levels. Quantitative variables were shown as mean \pm standard deviation and categorical variables were shown as percentage.

$P$ value $<0.05$ was considered as statistically significant.

\section{Results}

The present study included 79 RA patients for this research. In the first visit, 79 RA patients and on the other 5 visits, 73, 52, 33, 20, and 13 patients were evaluated, respectively. All 270 visits were evaluated. Female participants represented $51.9 \%$ and $48.1 \%$ were male. The mean age of all patients was $52.5 \pm 11.25$ years and was $52.26 \pm 12.5$ and $52.82 \pm 10.1$ years for men and women, respectively. The mean duration of the disease was $2.4 \pm$ 1.47 years. In the present study, $8.9 \%$ of patients were smokers, of which $66.7 \%$ and $83.3 \%$ were positive for RF and anti-CCP. Of these patients $78.48 \%$ had undergraduate education and $43 \%$ of the patients lived in rural areas (Table 1)

The RA patients presented with polyarthritis (77.21\%), oligoarthritis (15.18\%), and monoarthritis (1.26\%). Polyarthralgia was found in $6.32 \%$ of the patients. Diabetes, hypertension, ischemic heart disease, and hyperlipidemia were observed in the patients at 15.18 , $30.37,15.18$ and $17.72 \%$, respectively (Table 1 ).

Table 1. Demographic and clinical features of RA patients

\begin{tabular}{cc}
\hline Parameter & N (\%) \\
\hline Sex & Male: $38(48.1)$ \\
Marriage & Female: 41 (51.9 \\
& Married: $70(88.4)$ \\
Education & Miss data: $9(11.6)$ \\
& Under diploma: $62(78.48)$ \\
1 Rheumatry.ir web site is under supervision of the Rheumatology & Diploma $15(18.98)$ \\
Research Center in Shariati Hospital, Tehran Iran. & Post graduate: $2(2.53)$ \\
\end{tabular}




\begin{tabular}{|c|c|}
\hline Parameter & $\mathbf{N}(\%)$ \\
\hline Residence & $\begin{array}{l}\text { Urban: } 45(57) \\
\text { Rural: } 34(43)\end{array}$ \\
\hline Smoking & $\begin{array}{c}\text { Smoker: } 7(8.9) \\
\text { No smoker: } 63(79.7) \\
\text { Miss data: } 9(11.4)\end{array}$ \\
\hline History of rheumatism in first degree & $\begin{array}{l}\text { RA: } 9(11.39) \\
\text { SLE: } 2(2.53)\end{array}$ \\
\hline Positive history of disease & $\begin{array}{c}\text { Treated Tuberculosis: } 1(1.26) \\
\text { Treated Brucellosis: } 1 \text { (1.26) } \\
\text { Cerebrovascular accident (CVA): } 1 \text { (1.26) } \\
\text { Chronic kidney disease (CKD): } 2 \text { (2.53) } \\
\text { Poly arthritis: } 61 \text { (77.21) } \\
\text { Oligo arthritis: } 12(15.18) \\
\text { Mono arthritis: } 1(1.26) \\
\text { Poly arthralgia: } 5 \text { (6.32) }\end{array}$ \\
\hline Diabetes & $12(15.18)$ \\
\hline Hypertension & $24(30.37)$ \\
\hline Ischemic heart disease & $12(15.18)$ \\
\hline Hyperlipidemia & $14(17.72)$ \\
\hline Hypothyroid & $9(11.39)$ \\
\hline Post-partum relapse & $2(2.53)$ \\
\hline Knee arthroplasty & $2(2.53)$ \\
\hline
\end{tabular}

$\mathrm{RF}$ and anti-CCP was positive in RA patients at $58.22 \%$ and $62.02 \%$, respectively. High titer of RF on the first visit and all visits, thereafter, was seen in the patients at $25.31 \%$ and $32.91 \%$, respectively. High titer of anti-CCP on the first visit and total visits at $36.7 \%$ and $45.56 \%$, respectively, were seen in the patients. The mean of DAS, arthralgia, joint effusion, morning stiffness, joint tenderness, ESR, and CRP in the first to sixth visits are shown in Figure 1.
DAS

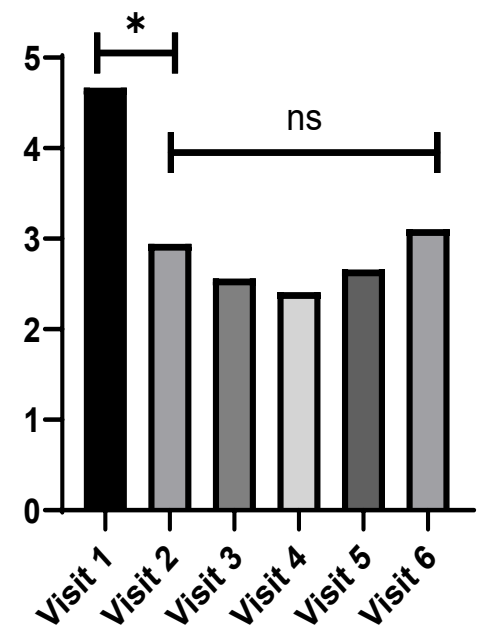

Arthralgia

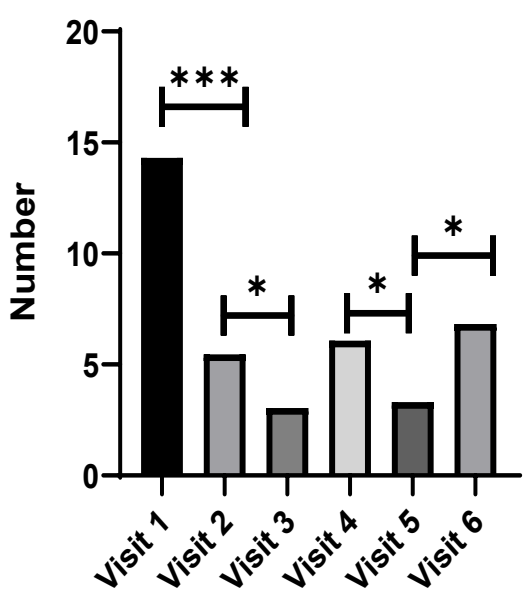

Joint efusion

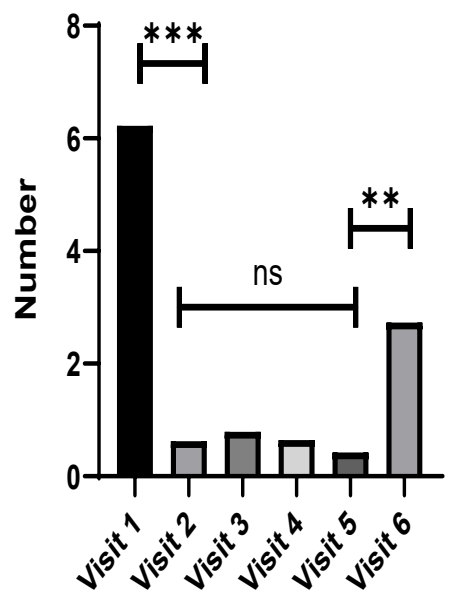




\section{Morning stiffness}

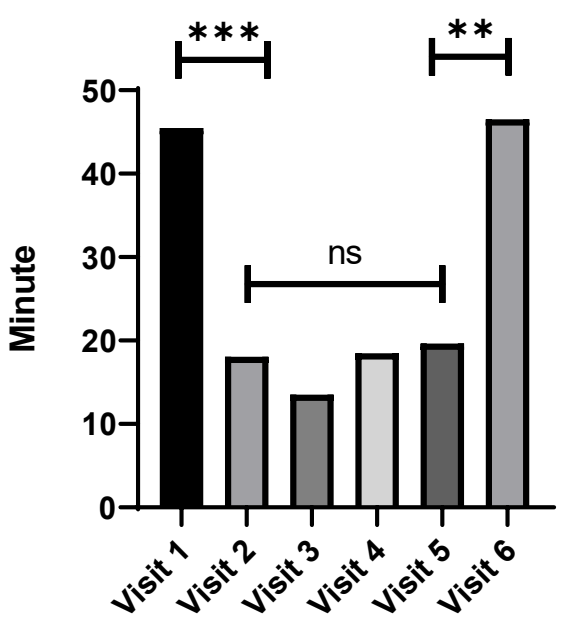

\section{Joint tender}

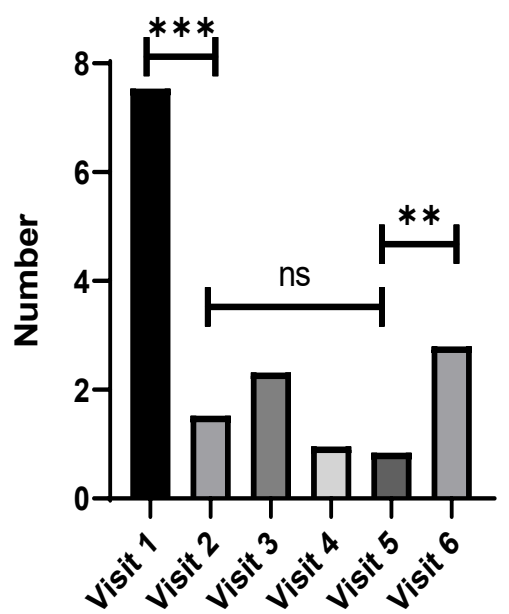

ESR

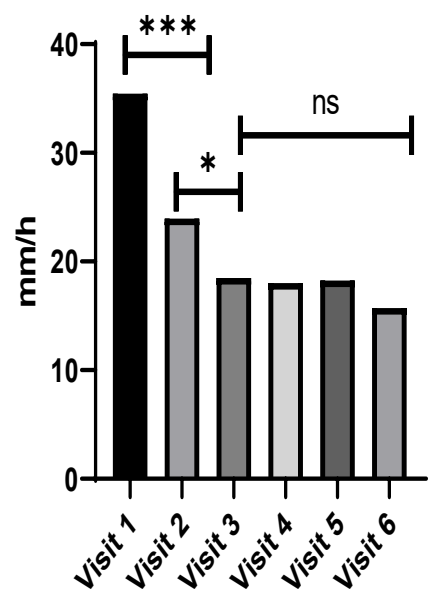

Figure 1. The mean of DAS, arthralgia, joint effusion, morning stiffness, joint tender, ESR and CRP in the first to sixth visits

The frequency of drugs used by patients on the first to sixth visits is shown in Table 2. Regarding extra-articular involvement, 10 cases $(12.65 \%)$ with pulmonary involvement were seen in one case of pleural effusion with positive anti-CCP, 4 cases of pulmonary fibrosis, and 5 cases of non-significant LAP (Table 3). Rheumatoid nodule was seen in $3.7 \%$ of the patients. Discontinuation of treatment due to drug side effects or arbitrary discontinuation is shown in Table 4. The most common cessation of treatment was due to the side effects of MTX, HCQ, and arbitrary discontinuation.

Table 2. Frequency of medications used by patients in the first to sixth visits.

\begin{tabular}{|c|c|c|c|c|c|c|c|c|c|c|}
\hline Visit & $\begin{array}{l}\text { Patient } \\
\text { (n) }\end{array}$ & PDN & HCQ & MTX & F.A & SSZ & LEF & Infliximab & Altebrel & Calcium-D \\
\hline 1 & 79 & 77 & 66 & 62 & 67 & 13 & 0 & 0 & 0 & 61 \\
\hline 2 & 73 & 68 & 60 & 62 & 65 & 16 & 2 & 0 & 2 & 60 \\
\hline 3 & 52 & 41 & 36 & 41 & 44 & 9 & 1 & 1 & 0 & 38 \\
\hline 4 & 33 & 26 & 17 & 21 & 25 & 8 & 1 & 1 & 0 & 24 \\
\hline 5 & 20 & 18 & 9 & 16 & 17 & 6 & 0 & 1 & 1 & 16 \\
\hline 6 & 13 & 8 & 5 & 10 & 10 & 2 & 0 & 0 & 1 & 0 \\
\hline
\end{tabular}

\begin{tabular}{ccccccccc}
\hline Visit & $\begin{array}{c}\text { Patient } \\
\text { (n) }\end{array}$ & $\begin{array}{c}\text { Vitamin } \\
\text { D3 }\end{array}$ & Alendronat & Cinnopar & Zolendoronat & Denozomab & NSAID & $\begin{array}{c}\text { Injection } \\
\text { to joint }\end{array}$ \\
\hline 1 & 79 & 39 & 21 & 1 & 1 & 0 & 17 & 7 \\
2 & 73 & 42 & 23 & 5 & 0 & 1 & 7 & 4 \\
3 & 52 & 29 & 16 & 0 & 1 & 0 & 4 & 0 \\
4 & 33 & 18 & 12 & 0 & 0 & 0 & 4 & 0 \\
5 & 20 & 9 & 6 & 1 & 0 & 0 & 2 & 1 \\
6 & 13 & 9 & 6 & 0 & 0 & 1 & 1 & 0 \\
\hline
\end{tabular}


Table 3. Frequency of extra-articular involvement

\begin{tabular}{lcc}
\hline & Parameter & N (\%) \\
\hline Pulmonary & Exudative pleural effusion & $1(1.26)$ \\
& Pulmonary fibrosis & $4(5.06)$ \\
& Non-significant LAP & $5(6.32)$ \\
Rheumatoid nodule & & $3(3.79)$ \\
\hline
\end{tabular}

Table 4. Discontinuation of treatment in RA patients

\begin{tabular}{lc}
\hline \multicolumn{1}{c}{ Parameter } & N (\%) \\
\hline MTX discontinue due to pregnancy & $3(3.79)$ \\
MTX discontinue due to raise of creatinine & $4(5.06)$ \\
MTX discontinue due to raise of liver enzymes > 2 folds & $4(5.06)$ \\
Reduction of MTX dose due to raise of liver enzymes 1- 2 folds & $4(5.06)$ \\
MTX discontinue due to pulmonary involvement & $3(3.79)$ \\
SSZ discontinue due to gastrointestinal intolerance & $3(3.79)$ \\
Altebrel discontinue due to intolerance & $2(2.53)$ \\
HCQ discontinue due to ocular complications & $7(8.86)$ \\
HCQ discontinue due to Pigmentation & $3(3.79)$ \\
HCQ discontinue due to vertigo & $1(1.26)$ \\
Arbitrary discontinue due to breastfeeding & $2(2.53)$ \\
Arbitrary discontinue & $16(20.25)$ \\
Discontinue treatment under the supervision of a physician & $2(2.53)$ \\
No data & $11(13.92)$ \\
\hline
\end{tabular}

The frequency of osteoarthritis (OA), osteopenia and osteoporosis in RA patients is shown in Table 5. Table 6 shows the frequency of admission, and foot and ankle arthritis. Evaluation of flare-up signs on the sixth visit of the RA patients was performed and the findings are presented in Table 7.

Table 5. The frequency of osteoarthritis (OA), osteopenia and osteoporosis in RA patients.

\begin{tabular}{cccccc}
\hline Visit & Knee OA & Hand OA & Hip OA & Osteopenia & Osteoporosis \\
\hline 1 & 17 & 15 & 0 & 13 & 8 \\
2 & 22 & 10 & 2 & 10 & 9 \\
3 & 11 & 5 & 1 & 4 & 5 \\
4 & 9 & 5 & 0 & 4 & 2 \\
5 & 6 & 4 & 1 & 4 & 1 \\
6 & 4 & 2 & 3 & 21 & 18
\end{tabular}


Table 6. Frequency of admission, foot arthritis and ankle arthritis in RA patients.

\begin{tabular}{ccc}
\hline Parameter & Frist visit & Total visits \\
\hline Admission & $14(17.72)$ & $1(7.04)$ \\
Foot arthritis & $6(7.59)$ & $10(3.7)$ \\
Ankle arthritis & $22(27.4)$ & $34(12.59)$ \\
\hline
\end{tabular}

Table 7. Evaluation of flare signs in sixth visit of RA patients $(\mathrm{N}=13)$

\begin{tabular}{cc}
\hline Parameter & N $(\mathbf{\%})$ \\
\hline Raise of arthralgia & $4(30.77)$ \\
Joint effusion & $3(23.07)$ \\
Joint tenderness & $3(23.07)$ \\
Raise of morning stiffness & $4(30.77)$ \\
\hline
\end{tabular}

\section{Discussion}

The aim of this study was to register and evaluate the demographic, clinical, and laboratory information of RA patients referred to Imam Khomeini Hospital, Tehran, Iran. The results of the present study showed that the mean age of the patients is 52 years. The minimum and maximum age of the patients were 24 and 83 years, respectively, which is consistent with the age range of men and women in previous studies, that is, 55-64 and 74-84 years. In a similar study by Eri Kato et al. [10], the mean age of RA patients was 55.8 years, and similar to the present study, no significant age range difference was observed between the ages of the men or of the women.

The female/male ratio was 1.06. Although RA was more prevalent among women, it is not in full agreement with the literature that the incidence of women is 2 to 3 times higher than that of men. In a similar study by Shangyi et al., $80.6 \%$ of RA patients in a single registry in China were female [11]. About $78 \%$ of patients had undergraduate education. Given that according to previous studies the disability of RA is higher in people with lower education, the need for education in these patients is more emphasized $[11,12]$.

Approximately $8.9 \%$ of the patients were smokers. The relative risk of RA is about 1.5 to 3.5 times higher in smokers, especially in women. The risks in smoking are exclusively related to RF and Anti-CCP positivity [13]. In the patients of the present study, about $66 \%$ and $83 \%$ of smokers were positive for RF and anti-CCP.

Cardiovascular risk factors in our patients were hypertension $(30 \%)$, hyperlipidemia $(17 \%)$ and diabetes $(15 \%)$, respectively, which reminds us to pay special attention to the appropriate treatment of these cases. Similar to these findings, in a study reported by Helga Radner et al., the prevalence of hypertension, hyperlipidemia, and diabetes in RA patients was $18.6 \%$, $6.2 \%$, and $9.9 \%$, respectively [14]. Given that cardiovascular causes are one of the most common causes of death in RA patients, control of RA disease, which causes a systemic inflammation and ultimately a risk factor for heart disease and control of other risk factors, is essential $[12,15]$.

Fifty-eight percent and $62 \%$ of the patients were RF and anti-CCP positive and 32.91 and $45.56 \%$ of RF and antiCCP patients had high titers. Considering that RF and anti$\mathrm{CCP}$ are progressive predictor factors in the patients and are related to the prognosis of the disease, so it is necessary to pay more attention to these cases $[12,16,17]$. In this regard, a similar study by Emil Rydell et al. [18] was performed on $223 \mathrm{RA}$ patients. The results of this study showed that smoking, RF, and anti-CCP significantly increased the risk of disease progression.

The mean ESR based on literature in RA patients is 50, which was an average of 35 in our patients. Subsequent visits show a decrease in ESR, which may indicate disease control along with other findings, including clinical examinations [17]. DAS-ESR was used to evaluate the activity of the disease, which averaged 4.6 at the initial visit and decreased to 2.6 at the fourth visit, which is defined as remission. However, there is an increase in DAS in visits 5 and 6, which can be due to a flare-up of RA. Despite the increase in DAS, it was still less than 3.2, which is defined as low disease activity [19, 20]. Kuriya B et al. [21] studied the DAS-ESR and DAS-CRP thresholds in 995 RA patients. Results of their study showed that the threshold values of DAS, based on CRP, are lower than DAS-ESR and the values of 2.5, 2.9 and 4.6 are considered as remission, low disease activity, and high disease activity, respectively.

Regarding extra-articular involvement, 10 cases $(12.65 \%)$ with pulmonary involvement were seen in one case of pleural effusion with positive anti-CCP, 4 cases of pulmonary fibrosis and 5 cases of non-significant LAP. In a study by Koduri et al. on 1,400 RA patients, the incidence of pulmonary involvement was 52 in 1,400 (3.71\%) in 
these patients [22]. Rheumatoid nodules were observed in $3.8 \%$ of the RA patients. In a study on 214 RA patients, Mirjana Ziemer et al. reported a prevalence of rheumatoid nodules of $27.5 \%$ [23]. Other extra-articular involvement, including ocular and cardiovascular involvement, was not seen in the patients in the present study.

HCQ discontinuation was seen in $8.8 \%$ of patients due to ocular complications. According to sources, the probability of retinal toxicity in the presence of risk factors such as consumption for more than ten years, doses higher than $6 \mathrm{mg} / \mathrm{kg}$, and renal failure of stage three and higher is about $7.5 \%$ in the first ten years of use and lack of risk factor is less than $2 \%$ [12]. In $3.79 \%$ of the cases skin pigmentation was observed due to HCQ complications. Rash and hyperpigmentation are common complications of HCQ that lead to discontinuation of treatment $[12,24]$.

An increase in liver enzymes more than two-fold was seen in $5 \%$ of the patients, which led to discontinuation of MTX treatment, and in about $5 \%$ of the patients there was an increase between 1-2 times normal, which was eliminated by reducing the dose of the drug. According to similar studies, the increase in liver enzymes above onefold normal is about $22 \%$ and more than twice is $1-2 \%$, which is more likely in the presence of other risk factors such as diabetes and obesity and the use of other hepatotoxic drugs $[12,25]$.

This study has potential limitations that should be noted. We had limited time to do research and collect data and lack of trained personnel for registration of RA was another limitation. Implementation of the registry system for rheumatic diseases in all rheumatology clinics in Iran is strongly suggested.

\section{Conclusion}

Clinical and laboratory data showed considerable disease control, however, relapse of disease was seen due to drug discontinuation. Disease registry makes it possible to record the necessary information of each patient, including demographic, clinical, paraclinical findings, and consultations giving the physician accurate access to patient information and unnecessary duplication of paraclinical examinations.

\section{Conflict of interest}

The authors report that they have no conflicts of interest to declare.

\section{References}

1. Humphreys JH, Verstappen SM, Hyrich KL, Chipping JR, Marshall T, Symmons DP. The incidence of rheumatoid arthritis in the UK: comparisons using the 2010 ACR/EULAR classification criteria and the 1987 ACR classification criteria. Results from the Norfolk Arthritis Register. Ann Rheum Dis 2013; 72(8):1315-20. doi: 10.1136/annrheumdis-2012-201960.

2. Koenders MI, van den Berg WB. Novel therapeutic targets in rheumatoid arthritis. Trends Pharmacol Sci 2015; 36(4):189-95. doi: 10.1016/j.tips.2015.02.001.

3. Singh JA, Saag KG, Bridges Jr SL, Akl EA, Bannuru RR, Sullivan MC. et al. 2015 American College of Rheumatology guideline for the treatment of rheumatoid arthritis. Arthritis Rheumatol 2016; 68(1):1-26. doi: 10.1002/art.39480.

4. McCarthy D, Mueller K, Wrenn J. Mayo Clinic: multidisciplinary teamwork, physician-led governance, and patient-centered culture drive world-class health care Commonwealth Fund New York; 2009.

5. Elkayam O, Pavelka K. Biologic registries in rheumatology: lessons learned and expectations for the future. Autoimmun $\operatorname{Rev}$ 2012; 12(2):329-36. doi: 10.1016/j.autrev.2012.05.009.

6. Daft RL, Lengel RH, Trevino LK. Message equivocality, media selection, and manager performance: Implications for information systems. MIS quarterly 1987:11(3):35566. doi.org/10.2307/248682
7. Mariette X, Gottenberg J-E, Ravaud P, Combe B. Registries in rheumatoid arthritis and autoimmune diseases: data from the French registries. Rheumatology (Oxford) 2011; 50(1):222-29. doi 10.1093/rheumatology/keq368.

8. Gottenberg J-E, Ravaud P, Cantagrel A, Combe B, Flipo $\mathrm{R}$, Schaeverbeke T. et al. Positivity for anti-cyclic citrullinated peptide is associated with a better response to abatacept: data from the 'Orencia and Rheumatoid Arthritis' registry. Ann Rheum Dis 2012; 71(11):1815-19. doi: 10.1136/annrheumdis-2011-201109.

9. Aletaha D, Neogi T, Silman AJ, Funovits J, Felson DT, Bingham III CO. et al. 2010 rheumatoid arthritis classification criteria: an American College of Rheumatology/European League Against Rheumatism collaborative initiative. Arthritis Rheum 2010; 62(9):2569-81. doi: 10.1002/art.27584.

10. Kato E, Sawada T, Tahara K, Hayashi H, Tago M, Mori H. et al. The age at onset of rheumatoid arthritis is increasing in Japan: a nationwide database study. Int J Rheum Dis 2017; 20(7):839-45. doi: 10.1111/1756-185X.12998.

11. Jin S, Li M, Fang Y, Li Q, Liu J, Duan X. et al. Chinese Registry of rheumatoid arthritis (CREDIT): II. prevalence and risk factors of major comorbidities in Chinese patients with rheumatoid arthritis. Arthritis Res Ther 2017; 19(1):251. doi: 10.1186/s13075-017-1457-z. 
12. Firestein GS, Budd RC, Gabriel SE, McInnes IB, O'Dell JR. Kelley and Firestein's textbook of rheumatology ebook. Elsevier Health Sciences; 2016.

13. Martinsson K, Johansson A, Kastbom A, Skogh T. Immunoglobulin (Ig) G1 and IgG4 anti-cyclic citrullinated peptide (CCP) associate with shared epitope, whereas IgG2 anti-CCP associates with smoking in patients with recent-onset rheumatoid arthritis (the Swedish TIRA project). Clin Exp Immunol 2017; 188(1):53-62. doi: 10.1111/cei.12901.

14. Radner H, Lesperance T, Accortt NA, Solomon DH. Incidence and prevalence of cardiovascular risk factors among patients with rheumatoid arthritis, psoriasis, or psoriatic arthritis. Arthritis Care Res (Hoboken) 2017; 69(10):1510-18. doi: 10.1002/acr.23171.

15. Blum A, Adawi M. Rheumatoid arthritis (RA) and cardiovascular disease. Autoimmun Rev 2019; 18(7):67990. doi: 10.1016/j.autrev.2019.05.005.

16. Van Venrooij WJ, Van Beers JJ, Pruijn GJ. Anti-CCP antibodies: the past, the present and the future. Nat Rev Rheumatol 2011; 7(7):391-98. doi: 10.1038/nrrheum.2011.76.

17. Birtane M, Yavuz S, Taştekin N. Laboratory evaluation in rheumatic diseases. World J Methodol 2017; 7(1):1-8. doi: 10.5662/wjm.v7.i1.1.

18. Rydell E, Forslind K, Nilsson J- $\AA$, Jacobsson LT, Turesson C. Smoking, body mass index, disease activity, and the risk of rapid radiographic progression in patients with early rheumatoid arthritis. Arthritis Res Ther 2018; 20(1):82. doi: 10.1186/s13075-018-1575-2.

19. Listing J, Kekow J, Manger B, Burmester G-R, Pattloch D, Zink A. et al. Mortality in rheumatoid arthritis: the impact of disease activity, treatment with glucocorticoids, TNFa inhibitors and rituximab. Ann Rheum Dis 2015; 74(2):415-21. doi: 10.1136/annrheumdis-2013-204021.
20. Putrik P, Ramiro S, Kvien TK, Sokka T, Pavlova M, Uhlig T. et al. Inequities in access to biologic and synthetic DMARDs across 46 European countries. Ann Rheum Dis 2014; 73(1):198-06. doi: 10.1136/annrheumdis-2012202603.

21. Kuriya B, Schieir O, Lin D, Xiong J, Pope J, Boire G. et al. Thresholds for the 28-joint disease activity score (DAS28) using C-reactive protein are lower compared to DAS28 using erythrocyte sedimentation rate in early rheumatoid arthritis. Clin Exp Rheumatol 2017; 35(5):799-03.

22. Koduri G, Norton S, Young A, Cox N, Davies P, Devlin J. et al. Interstitial lung disease has a poor prognosis in rheumatoid arthritis: results from an inception cohort. Rheumatology (Oxford) 2010; 49(8):1483-89. doi: 10.1093/rheumatology/keq035.

23. Ziemer M, Müller AK, Hein G, Oelzner P, Elsner P. Incidence and classification of cutaneous manifestations in rheumatoid arthritis. J Dtsch Dermatol Ges 2016; 14(12):1237-46. doi: 10.1111/ddg. 12680 .

24. Eljaaly K, Alireza KH, Alshehri S, Al-Tawfiq JA. Hydroxychloroquine safety: A meta-analysis of randomized controlled trials. Travel Med Infect Dis 2020; 36:101812. doi: 10.1016/j.tmaid.2020.101812.

25. Karlsson Sundbaum J, Eriksson N, Hallberg P, Lehto N, Wadelius M, Baecklund E. Methotrexate treatment in rheumatoid arthritis and elevated liver enzymes: A longterm follow-up of predictors, surveillance, and outcome in clinical practice. Int J Rheum Dis 2019; 22(7):1226-32. doi: 10.1111/1756-185X.13576. 